Received: 2021.07.01

Accepted: 2021.09.10 Available online: 2021.09 .23 Published: 2021.10.04

\title{
A Single-Center Study of a Resin Inlay Dental Implant-Fixed Prosthesis for Closing Proximal Contact Loss in 89 Patients Who Underwent 3-Year Follow-Up
}

Authors' Contribution: Study Design A Data Collection B Statistical Analysis C Data Interpretation D Manuscript Preparation E Literature Search F Funds Collection $G$

Corresponding Author: Financial support: Conflict of interest:
BCDEF 1 Qiyue Chen*

FG 1 Yong Shi*

EF 2 Zhaoqiang Zhang

AG 1 Guangbao Song
1 Special Clinics, Stomatological Hospital, Southern Medical University, Guangzhou, Guangdong, PR China

2 Department of Oral and Maxillofacial Surgery, Stomatological Hospital, Southern Medical University, Guangzhou, Guangdong, PR China

Background: Proximal contact loss (PCL) is very common between implant-fixed prostheses (IFPs) and adjacent teeth and leads to a high incidence of food impaction. Our aim was to determine if the mesio-distal adjustable (MDA) crown prostheses introduced in this study could easily establish new contacts intraorally without the intervention of prostheses retrieval.

Material/Methods: The MDA crown requires casting in the inlay framework at the proximal contact area. If PCL occurred, the resin in the inlay framework could be easily removed and refilled chairside. This single-center prospective study aimed to investigate the use of a resin inlay dental implant-fixed prosthesis for the closing proximal contact loss in 93 patients who were recruited from April 2017 to December 2017. Four patients dropped out during the 3-year follow-up; therefore, 89 patients were included. The effect of this technique was assessed by the implant mucosal status and follow-up investigations of food impaction.

Results: $\quad P C L$ occurred in $29.21 \%$ (26 prostheses) of the IFPs. The PCL rate at the mesial contact surfaces $(n=24,26.97 \%)$ was significantly higher than that at the distal contact surfaces $(n=5,8.33 \%)(P<0.01)$; however, the incidence of PCL was greater at adjacent teeth with significant bone resorption $(P=0.00)$. After readjusting the resin inlay and closing the clearance, implant mucosal status and food impaction were significantly reduced $(P<0.01)$.

Conclusions: The findings of this study showed that use of the MDA crown for closing PCL was time-saving and effective with satisfactory results at 3-year follow-up.

Keywords: Dental Care $\quad$ Dental Implants $\bullet$ Prostheses and Implants

Full-text PDF: https://www.medscimonit.com/abstract/index/idArt/933809

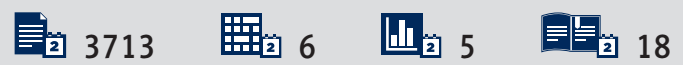




\section{Background}

Dental implant technology has become the main treatment for patients who are partially or totally edentulous [1]. With the extensive application of dental implant techniques in the clinic, postoperative complications from these techniques are receiving much attention. Recently, proximal contact loss (PCL) has been reported as a common post-treatment complication of implant-fixed prostheses (IFPs) [2-9], and $52.80 \%$ of open contacts with mesial aspects are 3.5 times more common than those with distal aspects [10].

Many studies have described methods for the prevention of $\mathrm{PCL}$, including expanding the adjacent interface of the prosthesis to increase the stability of the natural tooth position [11] and the use of a removable retainer to reduce the movement of the natural teeth [10]. However, the low strength of temporary adhesives can cause a prosthesis to fall off and even increase the risk of accidental swallowing [12].

When PCL occurs, the prosthesis must establish new contacts with the adjacent teeth, with the modification of either the teeth or prosthesis [13]. A retrievable prosthesis can be transferred to the dental laboratory, where porcelain is added to the proximal surface for closing the clearance. In contrast, the prosthesis placed with permanent adhesive is often difficult to remove. In addition, the stability of the abutment and implant can be adversely affected during the removal. Currently, many researchers recommend the use of screw-retaining prostheses for easy removal; however, screw retaining is not suitable in all cases. Also, traditional technical procedures are time-consuming, and the prostheses used for more than a year cannot be restored, even by adding porcelain on the proximal surface [6]. A chairside processing method has recently been described [14], in which a composite resin bonded to the implant-supported ceramic prosthesis is used to restore the proximal contact for the retrievable prosthesis. However, this method is not applicable to prostheses that are not retrievable.

Since the etiology of PCL remains unclear, this study focused on its treatment. We described a simple clinical method using a modified resin inlay to repair the PCL between the IFPs and adjacent teeth, which does not require that the prosthesis be retrievable. This single-center study investigated the use of a resin inlay dental implant-fixed prosthesis for closing proximal contact loss in 89 patients who underwent 3-year follow-up.

\section{Material and Methods}

\section{Patients}

This single-center prospective study enrolled 93 patients who had been treated with MDA crowns between April 2017 and
December 2017. Finally, 89 patients were included after 4 dropped out during the follow-up. All patients gave informed consent according to the ethical standards of the Institutional Review Board of the Stomatological Hospital, Southern Medical University, Guangzhou, China (no. 2017-01) and the 1964 Helsinki Declaration and its later amendments, or comparable guidelines. The patients were scheduled to undergo examinations immediately after implant insertion, with followup visits every 6 months until July 2020 in the Special Clinics, Stomatology of Southern Medical University, China (Figure 1). The study included 47 men and 42 women between the age of 31 and 77 years (mean age: $54.25 \pm 10.65$ years). The demographic characteristics of the patients are presented in Table 1.

\section{Patient and Public Sector Involvement}

Patients and the public sector were not involved in the design, conduct, reporting, or dissemination plans of this research study.

\section{Inclusion and Exclusion Criteria}

The inclusion criterion was patients with IFPs of the posterior teeth. Exclusion criteria were systemic illnesses, advanced periodontitis, and unwillingness to participate.

\section{Procedure of Resin Inlay Implant Prosthesis}

We designed this inlay implant prosthesis in early 2017 and named it the mesio-distal adjustable (MDA) crown. All prostheses involved in this study were made by the Guangdong Yuecheng Dental Technology Development Center, which was originally a Sino-foreign joint venture of Guangdong Yuecheng Dental Technology Development Co., Ltd., established by the Stomatological Hospital of Southern Medical University in 1992 with the introduction of foreign advanced technology, equipment, and management experience. The Guangdong Yuecheng Dental Technology Development Center passed the IS09001 quality certification test and obtained a medical device production license issued by the Guangdong Food and Drug Administration. In addition, all products have been registered with medical device certificates.

\section{Laboratory Procedure}

The width of the inlay was similar to the proximal surface but was not less than $2 \mathrm{~mm}$ (Figure 2A, 2B). To ensure sufficient resistance and the mechanical retention of form, the inlay framework was casted to resemble a class II cavity, with a thickness of $3 \mathrm{~mm}$ (Figure $2 \mathrm{C}$ ). The angle between the metal bottom wall and the side wall was between $90^{\circ}$ and $95^{\circ}$ (Figure 2D). The metal framework was filled with acrylic resin (SR Nexco Paste, Ivoclar Vivadent, Liechtenstein, Switzerland). The metal occlusal edge was kept $2 \mathrm{~mm}$ lower than the porcelain occlusal 


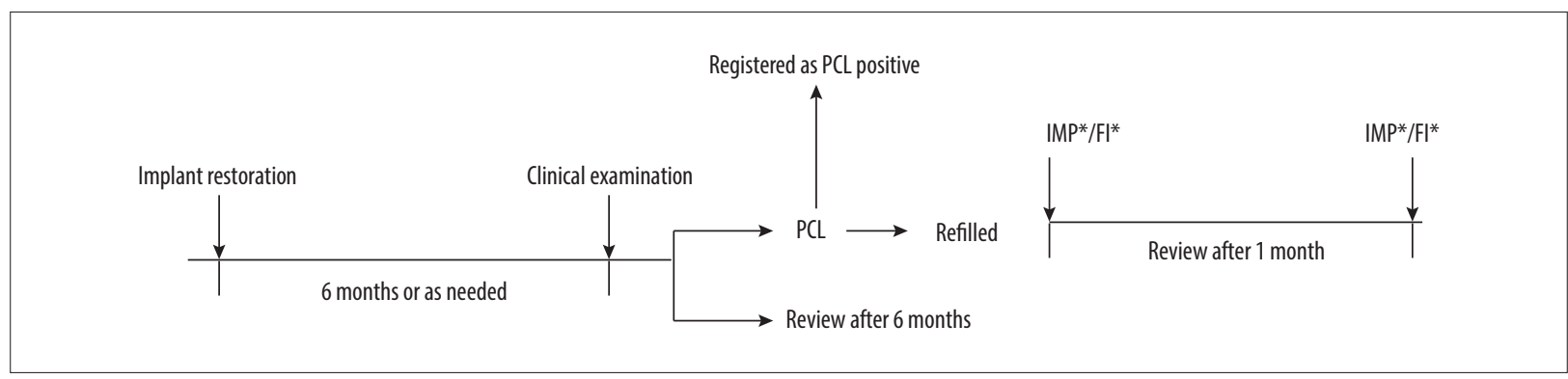

Figure 1. Timeline of interventions. IMP - implant mucosal bleeding on the probing index; FI score - the degree of food impaction.

Table 1. Patient characteristics.

\begin{tabular}{|c|c|c|c|c|}
\hline Item & $\mathbf{n}$ & PCL (n) & PCL rate $(\mathbf{n})$ & $P$ \\
\hline No. patients & 89 & 26 & 29.21 & \\
\hline \multicolumn{5}{|l|}{ Sex } \\
\hline Male & 47 & 10 & 21.28 & \multirow{2}{*}{0.08} \\
\hline Female & 42 & 16 & 38.10 & \\
\hline \multicolumn{5}{|l|}{ Age (years) } \\
\hline $30-44$ & 16 & 3 & 18.75 & \multirow{3}{*}{0.57} \\
\hline $45-60$ & 43 & 13 & 30.23 & \\
\hline $60+$ & 30 & 10 & 23.95 & \\
\hline Contact surfaces & 149 & 29 & 19.46 & \\
\hline Mesial & 89 & 24 & 26.97 & \multirow{2}{*}{$0.01^{*}$} \\
\hline Distal & 60 & 5 & 8.33 & \\
\hline \multicolumn{5}{|l|}{ State of IFP } \\
\hline Bounded & 60 & 18 & 30.00 & \multirow{2}{*}{0.81} \\
\hline Free-end & 29 & 8 & 27.59 & \\
\hline \multicolumn{5}{|l|}{ Jaw position } \\
\hline Maxilla & 33 & 8 & 24.24 & \multirow{2}{*}{0.43} \\
\hline Mandible & 56 & 18 & 32.14 & \\
\hline Bone support condition & 149 & & & \\
\hline $\operatorname{SBR}(\geq 1 / 2)$ & 45 & 18 & 40.00 & \multirow{2}{*}{$0.00^{\star}$} \\
\hline NSBR $(<1 / 2)$ & 104 & 11 & 10.58 & \\
\hline
\end{tabular}

SBR - significant bone resorption; NSBR - non-significant bone resorption; IFP - implant-fixed prostheses; PCL - proximal contact loss. Statistical significance was identified as alpha $=0.05$.

surface and was covered with acrylic resin material to obtain a better esthetic effect (Figure 2E). To support the biting force, the bottom wall of the resin was completely supported by metal (Figure 2F).

\section{Impact-Simulated Wear Test}

Six specimens each from the MDA and control groups were examined using a universal testing machine (ZQ-990b, Zhiqu, Dongguan, China). The materials were loaded on the machine with a 2.4-mm steel ball, perpendicular to the occlusal surface. Then the ball was lowered with a crosshead speed of 2 $\mathrm{mm} / \mathrm{min}$, and the maximum force required to produce fracture was recorded (Figure 3 ).

\section{Scanning Electron Microscopy}

Representative specimens from each group were analyzed. The analysis was performed using a scanning electron microscope (SEM, Nova NanoSEM450, FEI, USA). The coated surfaces were 


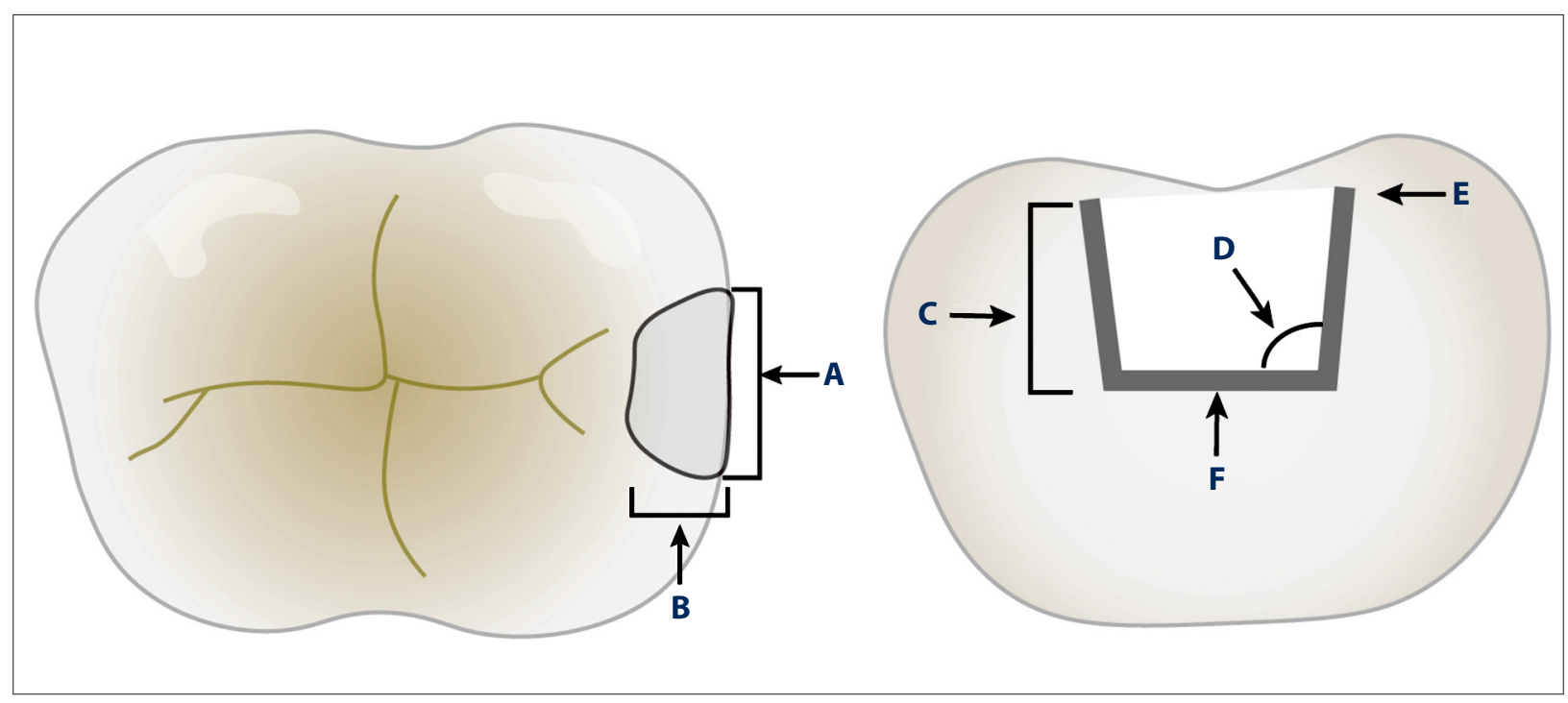

Figure 2. Schematic diagram of mesio-distal adjustable crown design. (A, B) The width of the inlay should not be less than $2 \mathrm{~mm}$. (C) The inlay framework should be a thickness of $3 \mathrm{~mm}$. (D) The angle should be $90^{\circ}$ to $95^{\circ}$. (E) The metal occlusal edge should be $2 \mathrm{~mm}$ lower than the porcelain occlusal surface. (F) The bottom wall of the resin must have complete support of the metal.

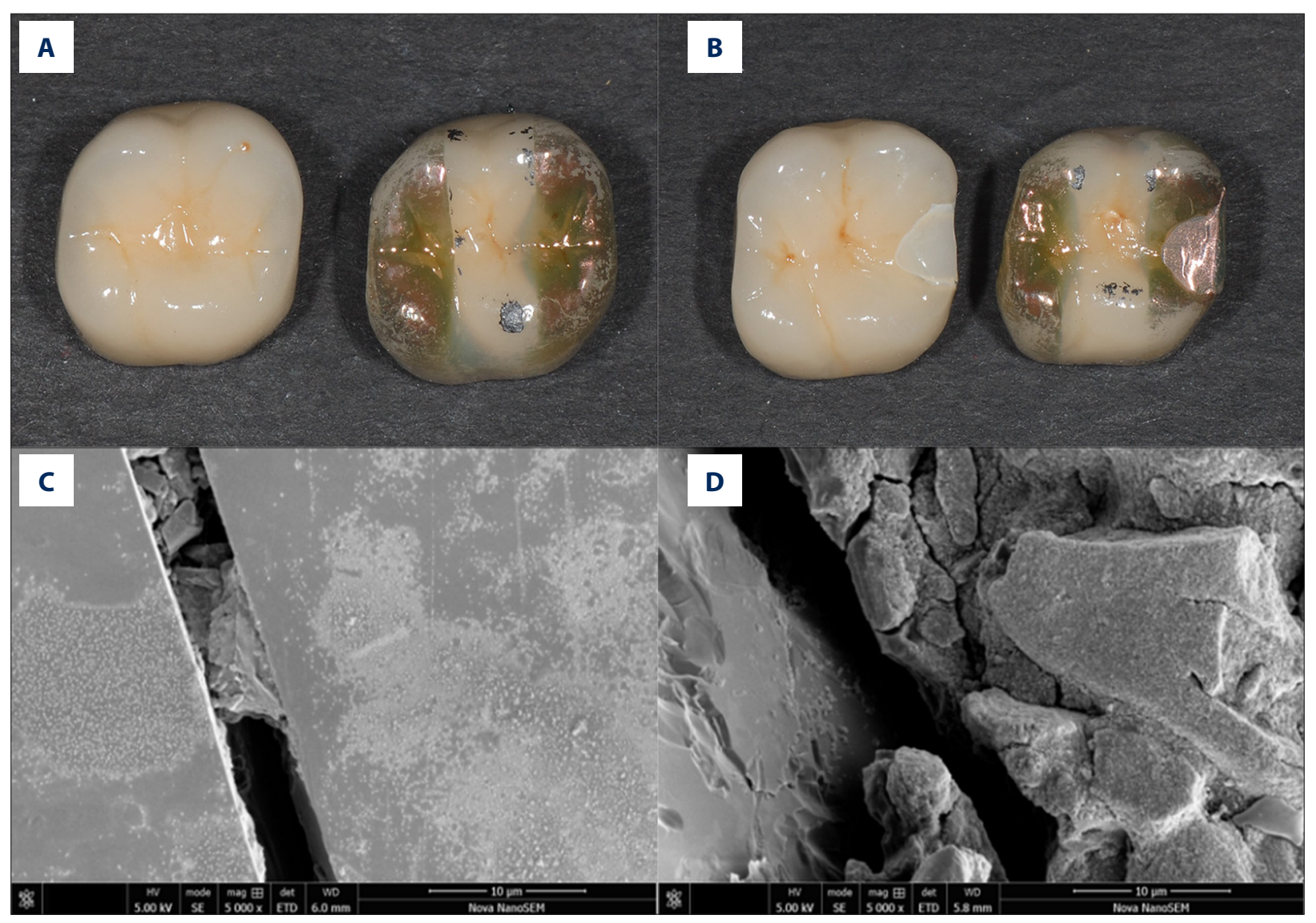

Figure 3. Impact-simulated wear test. (A, B) Representative images for the specimens before and after the impact-simulated wear test. (C, D) Scanning electron microscope images of impact-simulated wearing test from occlusal surfaces. The specimens were fractured and there was a lack of fragmenting powder in the fracture and the crack. (A, C) Representative images for the control group. (B-D) Representative images for the mesio-distal adjustable crown prosthesis group. 


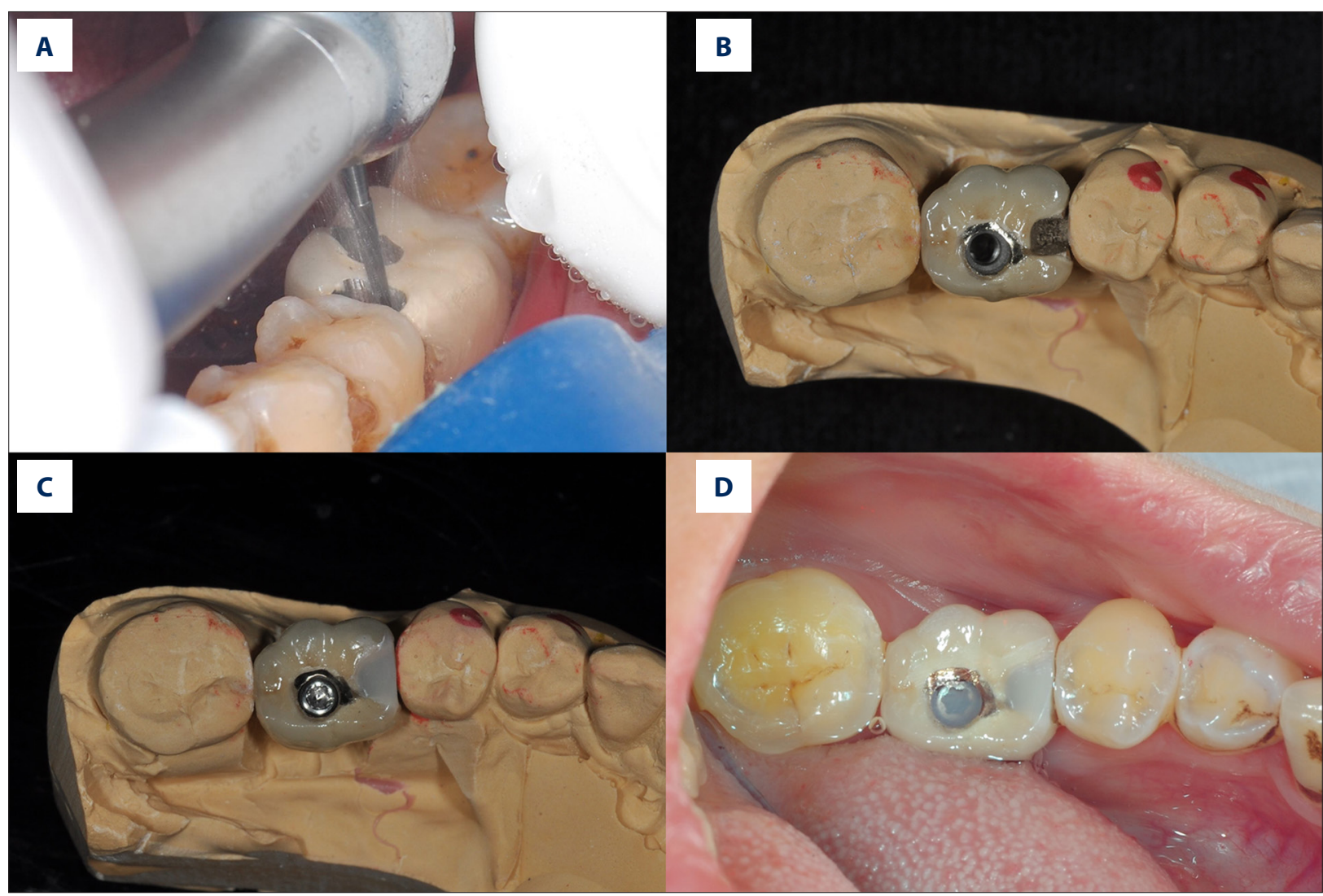

Figure 4. Prosthetic procedure and clinical procedure of mesio-distal adjustable (MDA) crown. (A) Wax model of the MDA crown. (B, C) Definitive prosthesis with mesial framework and screw access opening. (D) After removal of the healing abutment, note that the screw-retained prosthesis is seated in the implant.

visualized using a SEM with an operating voltage of $5 \mathrm{kV}$ and magnification of 5000x (Figure 3).

\section{Clinical Procedures}

Clinicians used light-curing composite resin (Filtek ${ }^{\text {TM }}$ Supreme Ultra Universal Restorative, 3M, USA) to fill or modify the proximal framework to establish the appropriate proximal contact. After adjustment, the prosthesis was seated intraorally with cement or was otherwise tightened with the screw (Figure 4).

\section{Maintenance Procedure}

Whenever the PCL was observed between the IFPs and the adjacent teeth on follow-up examinations, the resin in the inlay framework was easily removed and refilled with light-cured composite resin. The technique is unique, as the resin inlay created on the metal framework provides resistance to prevent resin fracture during functional loading. However, in the case of a cement-retained prosthesis, the resin inlay could be easily removed and refilled intraorally without requiring retrieval of the prosthesis (Figure 5). This technique provided a simple yet practical method for clinicians to repair PCL in IFPs. In this study, the implant prostheses were screw-retained. $\mathrm{PCL}$ and food impaction were observed for 3 years after the final placement of the prosthesis.

\section{Patients and Study Site}

\section{Prosthetic Procedure}

The Straumann (Straumann Group, Basel, Switzerland), Bego (Bego, Bremen, Germany), and Astra (Dentsply Sirona, Bensheim, Germany) implant systems were used in this study.

All IFPs were checked using dental floss $(50 \mu \mathrm{m})$ and were then flossed for high resistance during placement. The final prostheses were retained with glass ionomer cement (Fuji I, Japan). Cotton balls and temporary stopping were inserted into the abutment to cover the screw, whereas the opening of the crown was sealed with composite resin. The informed consent of each patient was obtained at the time of prosthesis placement. This design has been awarded Chinese national invention patents, no. ZL201710476873.3 and no. ZL201910220435.X. 


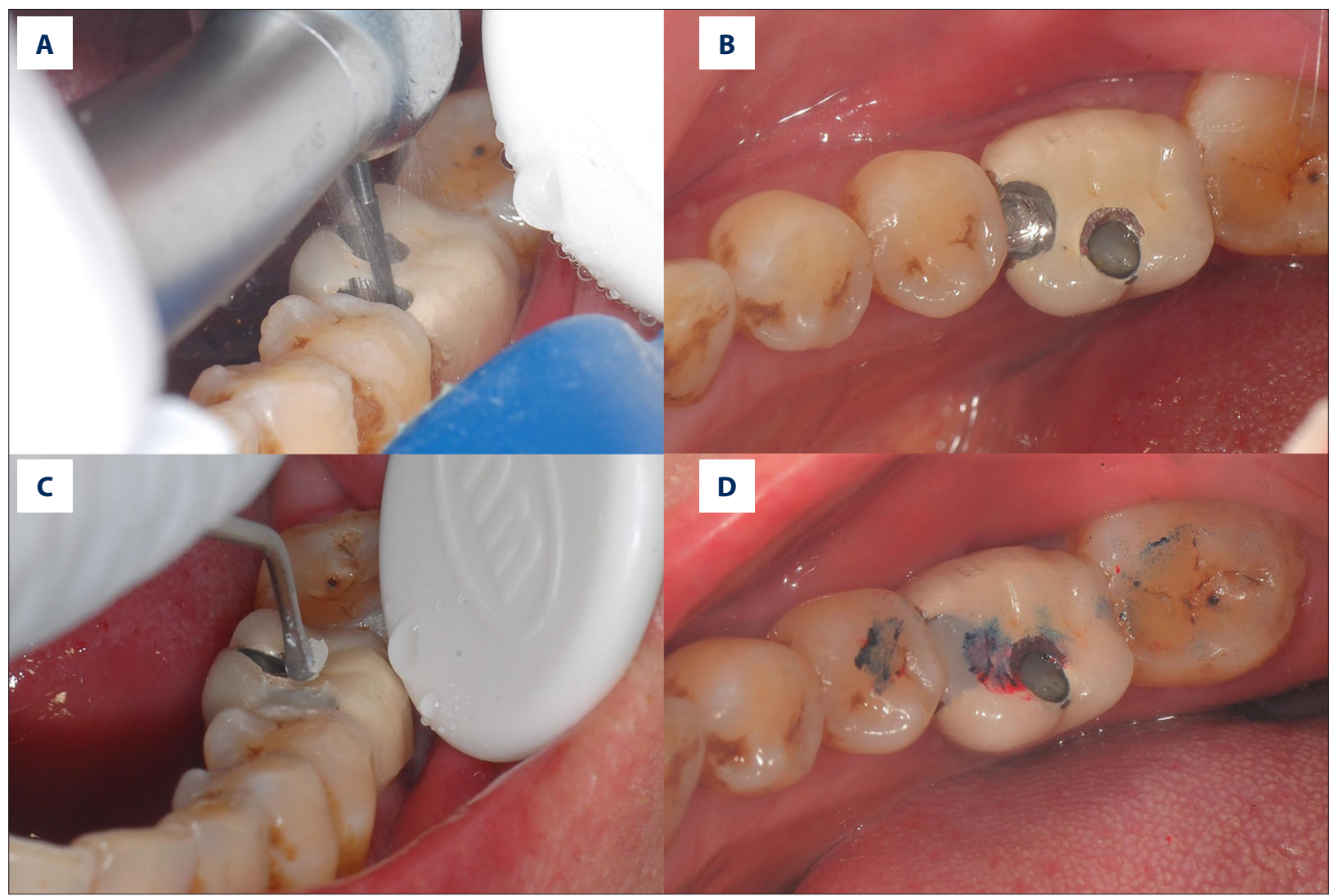

Figure 5. Treatment of proximal contact loss by mesio-distal adjustable crown. (A, B) Removing the original resin filling from the framework. (C, D) Refilling the framework with light-cured composite resin.

\section{Clinical Measurement}

Proximal contact tightness was evaluated during the immediate follow-up and every 6 months, or as needed, until July 2020. If the floss resistance was lower than normal, the proximal contact was considered an open contact. All measurements were performed by 1 prosthodontist and were further reviewed by 2 other prosthodontists. If at least 2 of the prosthodontists considered the prosthesis to have PCL, the case was recorded as positive and the time of $P C L$ occurrence was noted (Figure 1).

On placement of the prosthesis, a panoramic film was taken to evaluate the condition of the supporting bone. Because most patients were unwilling to undergo multiple X-rays, panoramic photography was used to ensure follow-up compliance. In the case of a patient having multiple prostheses, only 1 was selected for inclusion in the study. However, panoramic film was unable to distinguish the multiple levels of bone resorption of the adjacent teeth. If the level of bone support was no more than half of the root length, the bone support was considered to represent significant alveolar bone resorption; otherwise, it was considered as non-significant resorption.
Implant mucosal status was measured using controlled-force probing and evaluation of bleeding or suppuration. No bleeding was scored as 0 , single-point bleeding was scored as 1 , multi-point bleeding was scored as 2, and suppuration was scored as 3 (Table 2). After recording the implant-related mucosal bleeding on the probing (IMP) index with the degree of food impaction in implants with PCL, the clearance was closed by adjusting the resin fillings in the adjacent area. Meanwhile, patients were asked to estimate the degree of food impaction using the food impaction score. No food impaction was scored as 0 , occasional food impaction was scored as 1 , frequent food impaction was scored as 2, and apparent spontaneous pain was scored as 3 (Table 3 ). The IMP index and the food impaction score were reviewed after 1 month (Figure 1).

\section{Data Management and Statistical Analysis}

Because the impact-simulation wear test data were normally distributed, the $t$ test was used to analyze differences in fracture strength results between the groups.

Statistical analyses using the Wilcoxon symbol rank test were performed to estimate the effectiveness of this technique using the implant mucosal probing index and follow-up investigations 
Table 2. Implant mucosal bleeding on probing.

\begin{tabular}{cc|}
\hline IMP & \begin{tabular}{c} 
Bleeding on probing* \\
\hline 0
\end{tabular} \\
\hline 1 & No bleeding \\
\hline 2 & Single-point bleeding \\
\hline 3 & Multi-point bleeding \\
\hline
\end{tabular}

IMP - implant-related mucosal bleeding on the probing index. * Probing of 6 sites.
Table 3. Food impaction score.

\begin{tabular}{cc} 
Food impaction score (FIS) & $\begin{array}{c}\text { Subjective perception } \\
\text { No food impaction }\end{array}$ \\
\hline 0 & Occasional food impaction \\
\hline 1 & Frequent food impaction \\
\hline 3 & Apparent spontaneous pain* \\
\hline
\end{tabular}

* One patient was unable to clear the impaction and was forced to seek medical attention for apparent spontaneous pain.

Table 4. Impact-simulation wear test.

\begin{tabular}{cccccc} 
Group & $\mathbf{n}$ & Mean (SD) & \multicolumn{2}{c|}{$95 \%$ Confidence interval } \\
& & Lower bound & Upper bound & P \\
\hline MDA group & 6 & $980.80 \pm 15.02$ & -216.40 & 9.69 & 0.06 \\
\hline Control group & 6 & $1084.15 \pm 107.87$ & & \\
\hline
\end{tabular}

* Experimental group had the mesio-distal adjustable (MDA) crown.

on the reduction of food impaction. Whenever a prosthesis was placed, potential influencing factors were recorded to analyze the relative risk of $\mathrm{PCL}$ with the chi-squared test. All statistical analyses were conducted using SPSS version 20 (IBM Corp., Armonk, NY, USA). A $P$ value of $<0.05$ was used to indicate a statistically significant difference.

\section{Results}

\section{Impact-Simulation Wear Test and Scanning Electron Microscopy Observations}

The Levene test results revealed unequal variances between groups $(\mathrm{F}=16.627, P<0.05)$. The mean fracture strength results showed no significant differences between groups $(P>0.05)$. The MDA group had a lower mean fracture strength $(980.80 \pm 15.02$ $\mathrm{N})$ than the control group $(1084.15 \pm 107.87 \mathrm{~N})$, with a difference of -103.36 (95\% Cl, -216.40 to $9.69 ; t=-2.32 ; P=0.06$ ) (Table 4).

Figure 3 shows the representative scanning electron microscopy images for the MDA and control groups. The specimens were fractured with the lack of fragmenting powder in both the fractures and the cracks.

\section{PCL Was More Common in Mesial Aspects}

Of the 149 proximal contacts, 89 IFPs and 29 contact surfaces (26 prostheses) were observed to have contact loss. In agreement with previous studies, the PCL was more often mesial $(n=24,26.97 \%)$ than distal $(n=5,8.33 \%)(P<0.05)$ to an implant.

\section{PCL Relative to Bone and Support Level of Adjacent Teeth}

A total of 149 contact surfaces were observed in this study. During the follow-up period of approximately 3 years, the proportion of PCL with significant bone resorption of adjacent teeth was higher $(40.00 \%)$ than that of non-significant bone resorption of adjacent teeth (10.58\%). However, compared with the non-significant resorption, the relative risk of $\mathrm{PCL}$ with significant bone resorption of adjacent teeth was 3.78 (95\% Cl, 1.95-7.97).

\section{PCL and Implant Mucosal Inflammation}

We observed that $73.08 \%$ of the implants with PCL had implant-related mucosal inflammation (scores of 1 to 3), while the mucosal inflammation was reduced after the clearance was closed. At the open contact sites, fewer implants had an IMP score of $0(26.92 \%)$, whereas $46.15 \%$ had an IMP score of 1 , $23.08 \%$ had an IMP score of 2 , and $3.85 \%$ had an IMP score of 3. After clearance closing, more implants had an IMP score of 0 (84.61\%), but $11.54 \%$ had an IMP score of 1 , and $3.85 \%$ had an IMP score of 2 (Table 5). The paired Wilcoxon symbolic rank test further showed that the median IMP score of the subjects before treatment was 1 , while the median score after clearance closing was 0 . The Wilcoxon symbolic rank test showed $Z=-3.95(P=0.00)$, indicating that clearance closing was indeed helpful in reducing peri-implant inflammation. Inflammation in the surrounding prostheses with the most severe food impaction showed the most obvious reduction after clearance closure. 
Table 5. Proximal contact loss and corresponding impact-related mucosal bleeding on the probing index.

\begin{tabular}{|c|c|c|c|c|c|c|c|c|}
\hline & & 0 & & 1 & & 2 & & 3 \\
\hline Before & 7 & (26.92\%) & 12 & (46.15\%) & 6 & (23.08\%) & 1 & (3.85\%) \\
\hline After & 22 & (84.61\%) & 3 & (11.54\%) & 1 & (3.85\%) & 0 & \\
\hline
\end{tabular}

Table 6. Proximal contact loss and corresponding food impaction.

\begin{tabular}{|c|c|c|c|c|c|c|c|c|}
\hline & & 0 & & 1 & & 2 & & 3 \\
\hline Before & 5 & (19.23\%) & 14 & (53.85\%) & 5 & (19.23\%) & 2 & (7.69\%) \\
\hline After & 18 & (69.23\%) & 7 & (26.92\%) & 1 & (3.85\%) & 0 & \\
\hline
\end{tabular}

\section{PCL and Food Impaction}

When the patients with PCL were asked about food impaction, 5 patients reported they did not have food impaction, 14 reported that food impaction happened occasionally, 5 reported that food impaction occurred frequently, while 2 patients actively sought medical treatment because of pain caused by food impaction. After the clearance closing, 18 patients reported that they had no food impaction, 7 reported food impaction happened occasionally, and only 1 reported frequent food impaction (Table 6). Although food impaction did not disappear completely in all patients, it was reduced to varying degrees. PCL is one cause of food impaction. Other factors, such as occlusal trauma and occlusal contact position, alone or in combination, can also lead to vertical food impaction. The paired Wilcoxon symbolic rank test showed $Z=-4.16$ $(P=0.00)$, indicating that clearance closure was helpful in reducing food impaction.

\section{Complications}

The separation of the resin inlay from the framework ( 2 prostheses) and partial fracture of the resin inlay (1 prosthesis) were the only complications noted during the observation period.

\section{Discussion}

In this study, we showed that the MDA crown significantly reduced food impaction caused by PCL. This 3-year prospective study showed that the MDA crown had fewer complications than the control in clinical application. These results should be further validated by a large-cohort multi-center clinical study. Previously, with the traditional procedure, the occurrence of PCL required that the prosthesis establish new contacts with the adjacent teeth by modification of either the adjacent teeth or the prosthesis. Also, the traditional procedure is time-consuming, and prostheses used for more than 1 year cannot be restored by adding porcelain on the proximal surface. A chairside processing method has recently been described [14], in which a composite resin is bonded to the implant-supported ceramic prosthesis to restore the proximal contact; however, this requires a retrievable prosthesis. However, the MDA crown used in the present study can easily establish new contacts intraorally without requiring the retrieval of the prostheses. Moreover, this chairside procedure can save patients time and additional cost.

The etiology of PCL has not yet been determined. It is thought that physiological mesial drifting and alveolar bone resorption of the adjacent teeth are the most prominent etiological factors of PCL $[2,7]$. However, the presence of PCL in the distal aspects of a prosthesis cannot be justified by physiological mesial drifting; therefore, the etiology of PCL needs further investigation. Furthermore, wear and fractures on the edge of the proximal surface of the adjacent natural tooth/prosthesis due to high occlusal forces are also frequently observed. One study reported that food impaction occurs in approximately $40 \%$ of IFPs with PCL [8], and another reported that the incidence of food impaction was 2.2 times higher in a PCL group than in a control group [7]. These observations indicate that food impaction is closely related to PCL. Nonetheless, food impaction can create problems including the migration of teeth, dental caries, and periodontal issues [13].

In the present study, PCL was found to be mesial ( $n=24$, $26.97 \%$,) more often than distal $(n=5,8.33 \%)$ to an implant. This observation was consistent with other reported findings. Although several studies have suggested that mesial drifting of natural teeth is the main cause of $\mathrm{PCL}, \mathrm{PCL}$ also occurs at the distal aspect of prostheses, which cannot be explained by physiological mesial drifting. This suggests that $P C L$ is not caused by any single factor, although the specific origins are still unclear. In our study, after 3 years of follow-up observations, the incidence of PCL in the mesial aspect was observed to be 3 times higher than that in the distal aspect. 
Recently, the issue of food impaction caused by PCL has received increased attention. Unlike food lodgment, which can be removed by natural self-cleaning mechanisms, vertical food impaction causes gingivitis and peri-implantitis $[15,16]$. Also, the faulty contours and traumatic occlusion of PCL can result in vertical food impaction [17]. The present study observed that the occlusion trauma in adjacent teeth was related to chewing food impaction; resistance when using dental floss can be indicative of occlusion trauma. Occlusion trauma can occur during biting or chewing, where the displacement of adjacent teeth to the implant by the bite force might result in their separation from the prosthesis. Therefore, it is important to check the possibility of occlusion and abnormal displacement of the adjacent teeth when the prosthesis is placed, and especially while performing adjustments, to reduce the incidence of chewing food impaction.

A recent retrospective study of 4325 implants concluded that PCL can cause peri-implant mucositis [9]. The present study further showed that mucositis can be significantly reduced by closing the clearance $(P<0.01)$ and therefore reducing the amount of food impaction. Previous studies have shown that the incidence of PCL was higher in the elderly, which might be related to the increased mobility of the teeth in this age group $[2,8]$. Moreover, PCL has been found to be correlated with alveolar bone resorption [2], which is consistent with the results of the present study $(P<0.01)$. This observation suggests that the design of the MDA crown should be considered in cases in which the adjacent natural teeth show obvious alveolar bone resorption and are thus more likely to be easily displaced.

Food impaction occurs mainly in the posterior area. Therefore, the MDA crown is applicable to IFPs in the posterior area, especially in the molars. The inlay framework should have a depth of $3 \mathrm{~mm}$ to ensure the resistance and retention of the resin inlay. In cases of deeper framework, removal of the material inside and refill are often difficult. Moreover, the contact point of the adjacent teeth should still be in the middle to the occlusal third of the anatomical crown, even if the crown is extremely large due to gingival recession and periodontal disease. Since it is vertical food impaction that causes discomfort and damage to periodontal and dental tissue, extending the depth of the inlay framework is meaningless. However, the framework width should be at least $2 \mathrm{~mm}$ but not more than one-third of the mesio-distal dimension width, otherwise, it will increase the risk of porcelain collapse. The bottom wall of the resin should also be completely supported by the metal to support the biting force. Whenever the porcelain is piled up under the resin, it might collapse under the biting force. Eventually, it can also lead to partial fracture of the resin material because of the loss of the supporting wall. Therefore, the bottom edge of the framework should extend as far as possible to provide a greater area for adjustment of the resin if
PCL occurs in the future. The framework edge of the occlusal surface is generally hidden under the resin or porcelain layer and does not affect the esthetic appearance of the prosthesis.

While placing the prosthesis, the dentist should ensure that the prosthesis is well fitted and secure. The contact area of the adjacent surface can be adjusted chairside by either grinding or adding composite resin. Even though an increase in the contact area with the adjacent tooth can reduce the development of PCL, the occurrence of PCL can be accelerated if the proximal contact area remains too tight [18]. Meanwhile, $\mathrm{PCL}$ is a dynamic process that can occur many times with the same prosthesis. Compared with the contact area of traditional implant prostheses, the contact area of the MDA crown can be restored chairside and can also be re-adjusted several times thereafter. Because the PCL repair is simple and economical, it is more acceptable to patients than replacement with a new prosthesis. In the present study, cement retention was used with all the prostheses, which does not allow easy removal of the prosthesis. We also collected separate data on screw-retained MDA crown prostheses placed from 2018 (data not shown).

MDA crowns can have the mechanical complications of aging and loss of composite resin. After 3 years of clinical observation, we found that the main complication of MDA crowns was the shedding of resin filler from the framework with the partial defects of the marginal crest, which occurred in 3 prostheses. The resin filling is mechanically fixed in the inlay framework, so the casting requirements have a relatively high standard for the inlay framework. Thus, to address this issue, we contacted 3D digital printing companies to standardize the production and manufacturing of inlay framework plastic molds.

After making these initial clinical observations, we suggest that the most important indications for the MDA crown are the presence of loosening and significant bone resorption of the adjacent natural teeth. Thus, the design of the MDA crown allows for any possible modification in the future because the inlay framework can be adapted for use as part of a semi-fixed bridge.

The current study had some limitations. First, the study included only 89 patients who had been treated with MDA crowns between April 2017 and December 2017; therefore, a large multi-center study is needed for the validation of our results. Second, in younger patients ( $\leq 35$ years), the incidence of $P C L$ was low, and placement of porcelain in the inlay framework can be used. Based on our results and those of other studies, the incidence of $P C L$ is low in young patients and in patients with no bone absorption in adjacent teeth. Lastly, the complications associated with resin aging could have been avoided by using porcelain filling. Eventually, the porcelain can be removed when PCL occurs and filled with resin chairside. 


\section{Conclusions}

This study describes an inlay technique for closing the adjacent clearance in an implant-supported prosthesis. The findings from this study showed that a modified resin inlay dental implant-fixed prosthesis for closing proximal contact loss was time-saving and effective with satisfactory results at the 3-year follow-up. The advantages of this technique include the easy removal and refilling of a resin inlay on the prosthesis intraorally, without the retrieval of the prosthesis. In contrast to

\section{References:}

1. Muddugangadhar BC, Amarnath GS, Sonika R, et al. Meta-analysis of failure and survival rate of implant-supported single crowns, fixed partial denture, and implant tooth-supported prostheses. J Int Oral Health. 2015;7:11-17

2. Pang NS, Suh CS, Kim KD, et al. Prevalence of proximal contact loss between implant-supported fixed prostheses and adjacent natural teeth and its associated factors: A 7-year prospective study. Clin Oral Implants Res. 2017;28:1501-8

3. Jemt T. Measurements of tooth movements in relation to single-implant restorations during 16 years: A case report. Clin Implant Dent Relat Res. 2005;7:200-8

4. Wei $\mathrm{H}$, Tomotake $\mathrm{Y}$, Nagao K, Ichikawa T. Implant prostheses and adjacent tooth migration: Preliminary retrospective survey using 3-dimensional occlusal analysis. Int J Prosthodont. 2008;21:302-4

5. Koori H, Morimoto K, Tsukiyama Y, Koyano K. Statistical analysis of the diachronic loss of interproximal contact between fixed implant prostheses and adjacent teeth. Int J Prosthodont. 2010;23:535-40

6. Wat PY, Wong AT, Leung KC, Pow EH. Proximal contact loss between implant-supported prostheses and adjacent natural teeth: A clinical report. J Prosthet Dent. 2011;105:1-4

7. Byun SJ, Heo SM, Ahn SG, Chang M. Analysis of proximal contact loss between implant-supported fixed dental prostheses and adjacent teeth in relation to influential factors and effects. A cross-sectional study. Clin Oral Implants Res. 2015;26:709-14

8. Wong AT, Wat PY, Pow EH, Leung KC. Proximal contact loss between implant-supported prostheses and adjacent natural teeth: A retrospective study. Clin Oral Implants Res. 2015;26:e68-71 traditional prosthesis-modifying techniques done in the dental laboratory, the present chairside procedure can save the patient time and extra treatment cost.

\section{Declaration of Figures' Authenticity}

All the figures submitted have been created/generated by the authors. We further confirm that the images are original with no duplication and have not been previously published anywhere in whole or in part.

9. French $D$, Naito M, Linke B. Interproximal contact loss in a retrospective crosssectional study of 4325 implants: Distribution and incidence and the effect on bone loss and peri-implant soft tissue. J Prosthet Dent. 2019;122:108-14

10. Varthis S, Randi A, Tarnow DP. Prevalence of interproximal open contacts between single-implant restorations and adjacent teeth. Int J Oral Maxillofac Implants. 2016;31:1089-92

11. Sarig R, Lianopoulos NV, Hershkovitz I, Vardimon AD. The arrangement of the interproximal interfaces in the human permanent dentition. Clin Oral Investig. 2013;17:731-38

12. Rinke $S$, Roediger $M$, Eickholz $P$, et al. Technical and biological complications of single-molar implant restorations. Clin Oral Implants Res. 2015;26:1024-30

13. Greenstein G, Carpentieri J, Cavallaro J. Open contacts adjacent to dental implant restorations: Etiology, incidence, consequences, and correction. J Am Dent Assoc. 2016;147:28-34

14. Liu X, Liu J, Zhou J, Tan J. Closing open contacts adjacent to an implant-supported restoration. J Dent Sci. 2019;14:216-18

15. Jernberg GR, Bakdash MB, Keenan KM. Relationship between proximal tooth open contacts and periodontal disease. J Periodontol. 1983;54:529-33

16. Jeong JS, Chang M. Food impaction and periodontal/peri-implant tissue conditions in relation to the embrasure dimensions between implant-supported fixed dental prostheses and adjacent teeth: A cross-sectional study. J Periodontol. 2015:86:1314-20

17. Chopra A, Sivaraman K, Narayan Al, Balakrishnan D. Etiology and classification of food impaction around implants and implant-retained prosthesis. Clin Implant Dent Relat Res. 2019;21:391-97

18. Ren $\mathrm{S}$, Lin $Y$, Hu X, Wang Y. Changes in proximal contact tightness between fixed implant prostheses and adjacent teeth: A 1-year prospective study. J Prosthet Dent. 2016;115:437-40 